\title{
Determination of the B820 Subunit Size of a Bacterial Core Light-Harvesting Complex by Small-Angle Neutron Scattering ${ }^{\dagger}$
}

\author{
Zheng-Yu Wang,,$*$ Y Yoshiyuki Muraoka, ${ }^{\ddagger}$ Michihiro Nagao, ${ }^{\S}$ Mitsuhiro Shibayama, ${ }^{\S}$ Masayuki Kobayashi, ${ }^{\ddagger}$ and \\ Tsunenori Nozawa \\ Department of Biomolecular Engineering, Graduate School of Engineering, Tohoku University, Sendai 980-8579, Japan, and \\ Neutron Scattering Laboratory, Institute for Solid State Physics, University of Tokyo, 106-1 Shirakata, Tokai 319-1106, Japan
}

Received March 18, 2003; Revised Manuscript Received May 27, 2003

\begin{abstract}
The B820 subunit is an integral pigment-membrane protein complex and can be obtained by both dissociation of the core light-harvesting complex (LH1) in photosynthetic bacteria and reconstitution from its component parts in the presence of $n$-octyl $\beta$-D-glucopyranoside (OG). Intrinsic size of the B820 subunit from Rhodospirillum rubrum LH1 complex was measured by small-angle neutron scattering in perdeuterated OG solution and evaluated by Guinier analysis. Both the B820 subunits prepared by dissociation of LH1 and reconstitution from apopolypeptides and pigments were shown to have a molecular weight of $11400 \pm 500$ and radius of gyration of $11.0 \pm 1.0 \AA$, corresponding to a heterodimer consisting of one pair of $\alpha \beta$-polypeptides and two bacteriochlorophyll $a$ molecules. Molecular weights of micelles formed by $\mathrm{OG}$ alone in solutions were determined in a range from 30000 to 50000 over concentrations of $1-5 \%(\mathrm{w} / \mathrm{v})$, and thus are much larger than that of the B820 subunit. Similar measurement on the pigment-depleted apopolypeptides revealed highly heterogeneous behavior in the OG solutions, indicating that aggregates with various sizes were formed. The result provides evidence that bacteriochlorophyll $a$ molecules play a crucial role in stabilizing and maintaining the B820 subunits in the dimeric state in solution. Further measurements on individual $\alpha$ - and $\beta$-polypeptides exhibited a marked difference in aggregation property between the two polypeptides. The $\alpha$-polypeptides appear to be uniformly dissolved in OG solution in a monomeric form, whereas the $\beta$-polypeptides favor a self-associated form and tend to form large aggregates even in the presence of detergent. The difference in aggregation tendency was discussed in relation to the different behavior between $\alpha$ - and $\beta$-polypeptides in reconstitution with bacteriochlorophyll $a$ molecules.
\end{abstract}

Despite intense interest and continuing investigation, controversy over the nature of an intermediate form of bacterial core light-harvesting complexes $\left(\mathrm{LH} 1^{1}\right)$ has not been settled. The LH1 complex, serving as highly efficient molecular machinery for collection and transfer of solar energy to photochemical reaction centers in photosynthetic bacteria, is a large multimer of a minimal unit composed of two small integral membrane polypeptides ( $\alpha$ and $\beta$, ca. 6 $\mathrm{kDa})$ associated with two bacteriochlorophyll (BChl) and one carotenoid molecule. Upon addition of detergents, the LH1 multimer can be dissociated into a structural subunit characterized by a $Q_{y}$ absorption band at $820 \mathrm{~nm}(1)$. Both the

† This work was supported by Grant-in-aid for Scientific Research (No. 12878108 and 14550784), a Grant-in-Aid for the COE Project "Giant Molecules and Complex Systems", and Scientific Research of Priority Areas: Single-Cell Molecular Technology (No. 11227203), the Ministry of Education, Science, Sports and Culture, Japan.

* Corresponding author. Fax: +81-22-217-7278. E-mail: wang@ biophys.che.tohoku.ac.jp.

$\$$ Tohoku University.

$\S$ University of Tokyo.

${ }^{1}$ Abbreviations: BChl, bacteriochlorophyll; LH1, core light-harvesting; LH2, peripheral light-harvesting; B820 and B777, core lightharvesting complex subunits absorbing at 820 and $777 \mathrm{~nm}$; HPLC, high performance liquid chromatography; OG, $n$-octyl $\beta$-D-glucopyranoside; Rps, Rhodopseudomonas; Rsp, Rhodospirillum; SANS, small-angle neutron scattering. subunit (referred to as B820) and LH1 complex can be reversibly dissociated and reconstituted from the their components (2). Early chromatographic studies by gel filtration estimated a range of molecular weight from 32 to $65 \mathrm{kDa}$ for the $\mathrm{B} 820$ subunit, roughly corresponding to a tetramer composed of two minimal structural units $(1,3)$. In contrast, spectroscopic evidence suggested that the B820 is a heterodimer consisting of two interacting $\mathrm{BChl} a$ molecules linked to one $\alpha \beta$-polypeptide pair $(4,5)$. Recently, identity of the B820 has been reexamined with equilibrium between several dissociated and oligomeric forms (6-8). Pandit et al. showed that the association of monomeric B777 into B820 is a dimeric reaction (6), whereas Arluison et al. demonstrated that the association form of the B820 may depend on the concentration of octylglucoside detergent $(n$ octyl $\beta$-D-glucopyranoside, OG), i.e. at low OG concentration the $\mathrm{B} 820$ is probably a dimer, but at higher OG concentration $(>1.2 \%)$ the B820 may be a tetramer (7).

All the methods mentioned above have their inherent problems and are indirect for estimating the intrinsic size of an integral membrane protein complex embedded in detergent micelles. More direct approaches would inevitably need to distinguish between the internal membrane protein and the detergent shell. The number of the detergent molecules attached to the membrane protein is considered to depend 
on both the protein and detergent concentrations. One of such techniques is the use of small-angle X-ray or neutron scattering for evaluation of the protein size in solution. Due to a large difference in neutron coherent scattering length between hydrogen and deuterium, the neutron scattering is more powerful, compared with X-ray, for identifying the scattering from selected components within a macromolecular membrane protein assembly by using combinations of deuterated and nondeuterated solvents, detergents, and proteins (9). The small-angle neutron scattering (SANS) provides information on size, molecular weight, shape, volume of macromolecules, and internal packing for multicomponent protein complexes and has been successfully applied for the characterization of various photosynthetic complexes (10-12). Because neutron scattering length density of fully deuterated $\mathrm{OG}$ is almost identical with that of $\mathrm{D}_{2} \mathrm{O}$, the effects of detergent can be efficiently removed, and as a result the neutron scattering from membrane proteins can be selectively detected by using the perdeuterated OG in $\mathrm{D}_{2} \mathrm{O}$ solution. In this study, we present results of the SANS measurements on both the B820 subunits obtained by dissociation of LH1 complex and reconstitution from apopolypeptides and BChl $a$ molecules in perdeuterated OG solution. Highly concentrated samples $(\sim 1 \mathrm{mM})$ were used in the experiments. For comparison, similar measurements were also performed on pigment-depleted apopolypeptides, individual $\alpha$ - and $\beta$-polypeptides under the same experimental condition. Physical sizes and molecular weights were determined by Guinier analysis without introducing any fitting parameters. Because structural homogeneity is essential for conducting such experiment at high concentrations, we have employed a novel reconstitution method for producing highly homogeneous and concentrated B820 subunits (13), as can be judged from absorption spectra and highresolution nuclear magnetic resonance spectroscopy.

\section{MATERIALS AND METHODS}

Preparation of B820 Subunits, Apopolypeptides, $\alpha$ - and $\beta$-Polypeptides. Isolation and purification of LH1 complex from wild-type photosynthetic purple bacterium Rhodospirillum (Rsp.) rubrum were described elsewhere $(13,14)$. For the preparation of intact B820 subunit, the LH1 complex was first extracted with benzene to remove carotenoids. The lyophilized carotenoid-depleted LH1 powder was dissolved in phosphate buffer $\left(\mathrm{D}_{2} \mathrm{O}, \mathrm{D}>99.9 \%, 50 \mathrm{mM}, \mathrm{pD} 7.0\right)$ containing $4 \%(\mathrm{w} / \mathrm{v})$ perdeuterated OG- $d_{28}(\mathrm{D}>97 \%$, Anatrace Inc., Ohio), followed by thorough mixing. Unless otherwise stated, the perdeuterated OG-containing phosphate buffer was used throughout the SANS experiments. Reconstituted B820 subunit was prepared using a method described previously (13), except that natural abundance BChl $a$ and $4 \%$ perdeuterated OG- $d_{28}$ solution were employed in this study. Apopolypeptides were obtained by repeatedly extracting the carotenoid and BChl $a$ molecules from LH1 complex with benzene and methanol. The pigment-depleted apopolypeptides were dissolved in the perdeuterated OGcontaining phosphate buffer. The $\alpha$ - and $\beta$-polypeptides were separated from the apopolypeptides by HPLC on a reversephase column (TSKgel, ODS-80Ts, $21.5 \mathrm{~mm} \times 30 \mathrm{~cm}$, $\mathrm{TOSOH})$. To remove large aggregates in the solutions, all samples were centrifuged at $14000 \mathrm{~g}$ for $10 \mathrm{~min}$ before measurements. Precipitates were washed with distilled water several times and then were dried for weighing. After corrections, the final concentrations of intact B820, reconstituted B820, apopolypeptide, and $\alpha$ - and $\beta$-polypeptides were $9.4,12.2,12.8,16.6$, and and $6.7 \mathrm{mg} / \mathrm{mL}$, respectively. Nondeuterated OG was from Dojindo Laboratories (purity: $>98 \%$ ) and was used for measurements of micelle size in solution. Absorption spectra were recorded on a Beckman DU-640 spectrophotometer, using an ultrathin cuvette $(0.105$ $\mathrm{mm}$ path length) for the highly concentrated B820 subunits.

SANS Measurements. SANS experiments were performed on the instrument (SANS-U) installed in JRR-3M of Japan Atomic Energy Research Institute (JAERI). The neutron beam from a cold source was monochromatized by a velocity selector (Dornier). Scattered neutrons were led to an evacuated scattering flight tube and recorded using a twodimensional position-sensitive detector (Ordela) with an active area of $65 \times 65 \mathrm{~cm}^{2}$ and resolution of $5 \times 5 \mathrm{~mm}^{2}$. The 2D data were radially averaged and converted as a function of $q=4 \pi \sin \theta / \lambda$, where $\theta$ is half the scattering angle and $\lambda$ is the neutron wavelength (7.0 $\AA$ ). Sample-todetector distances were either 2 or $4 \mathrm{~m}$ depending on the samples. Details on specification of the instrument were given elsewhere (11). The raw data were corrected for solvent background, transmission and incoherent scattering, and finally were normalized to absolute intensity units $\left(\mathrm{cm}^{-1}\right)$ using Lupolen (15) or the incoherent scattering of $\mathrm{H}_{2} \mathrm{O}$ (1 mm path length) (16) as references. The sample solutions were placed in a quartz cell of $4 \mathrm{~mm}$ path length, and measurements were conducted at room temperature $\left(22^{\circ} \mathrm{C}\right)$.

SANS data Analysis. The SANS data were analyzed by standard Guinier method (17), i.e., the scattered intensity is expected to vary as

$$
I(q)=I(0) \exp \left(-R_{\mathrm{g}}^{2} q^{2} / 3\right)
$$

in the region of $q R_{\mathrm{g}} \leq 1.3$. Slope of the plot, $\ln I(q)$ vs $q^{2}$, yields the radius of gyration $R_{\mathrm{g}}$ of the complexes, which is defined as the root-mean-squared distance of all the atoms to the centroid of the scattering length distribution and therefore is characteristic of the relative size of complexes. The $y$-intercept yields $I(0)$, the scattering intensity at $0^{\circ}$ angle. $I(0)$ contains information on molecular weight of the complexes and can be expressed by the following formula (16):

$$
\frac{I(0)_{\mathrm{s}}}{I(0)_{\mathrm{w}}}=f \frac{4 \pi T_{\mathrm{s}}}{\left(1-T_{\mathrm{w}}\right)} M_{\mathrm{r}} N_{\mathrm{A}} t \times 10^{3}\left[\frac{\sum b_{i}}{\left(M_{\mathrm{r}}\right)_{\mathrm{m}}}-\frac{\bar{b} \bar{v}}{N_{\mathrm{A}}}\right]^{2}
$$

Here, $I(0)_{\mathrm{s}}$ and $I(0)_{\mathrm{w}}$ represent forward scatterings of sample and $\mathrm{H}_{2} \mathrm{O}$, respectively; $f$ is a correction factor depending on the neutron wavelength $(f=0.86$ for $\lambda=7.0 \AA) ; T_{\mathrm{s}}$ and $T_{\mathrm{w}}$ are transmissions of sample and $\mathrm{H}_{2} \mathrm{O}$, respectively; $M_{\mathrm{r}}$ is the molecular weight of the whole complex; $N_{\mathrm{A}}$ is Avogadro's number; $t$ is the path length of the sample cuvette in $\mathrm{cm}$; $\Sigma b_{i}$ is the sum of the neutron scattering lengths of the atoms in a minimal unit of the complex; $\left(M_{\mathrm{r}}\right)_{\mathrm{m}}$ is the molecular weight of the minimal unit of the complex and can be calculated on the basis of amino acid sequence; $\bar{b}$ is the mean neutron scattering length density of the buffer; and $\bar{v}$ is the partial specific volume of the complex. We estimated that $70 \%$ of the exchangeable protons equilibrate with the solvent 


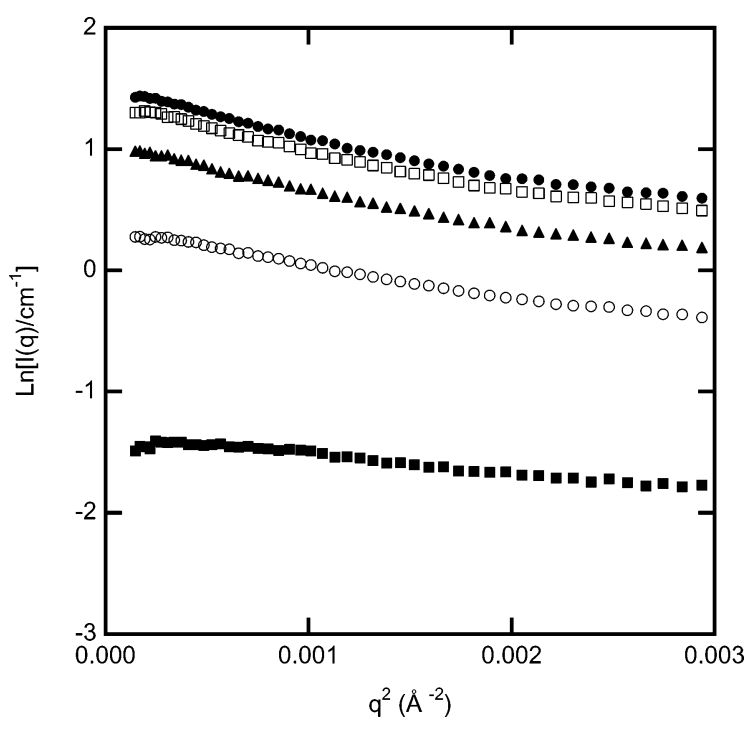

FIGURE 1: Guinier plots of SANS profiles for the nondeuterated OG micelles in $50 \mathrm{mM}$ phosphate buffer $\left(\mathrm{D}_{2} \mathrm{O}, \mathrm{pD}\right.$ 7.0). Symbols: 口, $1 \%(\mathrm{w} / \mathrm{v}) ; \mathrm{O}, 2 \%(\mathrm{w} / \mathrm{v}) ; \boldsymbol{\Delta}, 3 \%(\mathrm{w} / \mathrm{v}) ; \square, 4 \%(\mathrm{w} / \mathrm{v}) ; \boldsymbol{0}, 5 \%$ $(\mathrm{w} / \mathrm{v})$.

Table 1: Sizes and Aggregation Numbers $(N)$ of OG Micelles Determined from SANS at Various Concentrations in $50 \mathrm{mM}$ Phosphate Buffer $\left(\mathrm{D}_{2} \mathrm{O}, \mathrm{pD} 7.0\right)$

\begin{tabular}{crccc}
\hline$\%(\mathrm{w} / \mathrm{v})$ & $\mathrm{mM}$ & $R_{\mathrm{g}}(\AA)$ & $M_{\mathrm{r}}$ & $\mathrm{N}$ \\
\hline 1 & 34 & 22.1 & 30950 & 106 \\
2 & 68 & 30.2 & 44380 & 152 \\
3 & 102 & 33.5 & 50520 & 173 \\
4 & 136 & 35.4 & 50220 & 172 \\
5 & 170 & 36.2 & 44670 & 153 \\
\hline
\end{tabular}

on the basis of the result of $\mathrm{H}-\mathrm{D}$ exchange measurement by two-dimensional ${ }^{1} \mathrm{H}-{ }^{15} \mathrm{~N}$ heteronuclear single quantum coherence NMR spectroscopy (data not shown).

\section{RESULTS}

Estimation of the Micelle Sizes of OG Alone at High Concentrations. Before we describe the SANS result of $\mathrm{B} 820$, it is useful to reexamine the size of micelles formed by $\mathrm{OG}$ alone in solutions because there has been a relatively large variance reported in the literature and little information is available for the concentrated solutions as in the case of this study. Figure 1 shows the Guinier plots of SANS profiles for the nondeuterated $\mathrm{OG}$ in $50 \mathrm{mM}$ phosphate buffer $\left(\mathrm{D}_{2} \mathrm{O}\right.$, pD7.0) at different OG concentrations. Due to the large sizes of micelles, SANS measurements were conducted with a sample-to-detector distance of $4 \mathrm{~m}$. For all samples, the Guinier plots can be fitted by straight lines in the smallangle region, indicating that the OG micelles were homogeneous over the wide concentration range. Table 1 summarizes the values of radius of gyration, molecular weight, and aggregation number of the OG micelles. The radius of gyration increased most drastically as $\mathrm{OG}$ concentration increased from 1 to $3 \%(\mathrm{w} / \mathrm{v})$, followed by a small increase at concentrations higher than $3 \%(\mathrm{w} / \mathrm{v})$. The value of molecular weight reached a maximum at $\mathrm{OG}$ concentrations around $3-4 \%(\mathrm{w} / \mathrm{v})$. The aggregation numbers were calculated from the corresponding molecular weights. Our results show that OG micelles in the very concentrated solutions have radii of gyration of about 33 to $36 \AA$ and molecular weights of 45000 to 50000 . The sizes of OG micelles determined from

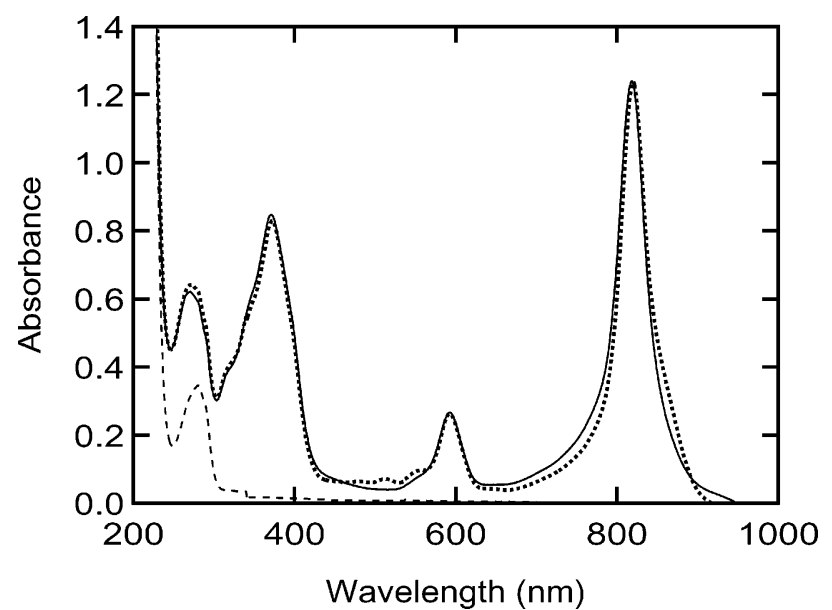

FIGURE 2: Absorption spectra of the highly concentrated B820 subunits and apopolypeptides used for the SANS measurements. The B820 subunits were prepared by dissociation of carotenoiddepleted LH1 complex $(9.4 \mathrm{mg} / \mathrm{mL}$, dotted line) and reconstitution from apopolypeptides and BChl $a(12.2 \mathrm{mg} / \mathrm{mL}$, solid line) at OG$d_{28}$ concentration of $4 \%(\mathrm{w} / \mathrm{v})$. Absorption spectrum of the intact B820 subunit was adjusted to have the same intensity as that of reconstituted B820 subunit at $819 \mathrm{~nm}$. The absorption spectrum for the apopolypeptides $(16.1 \mathrm{mg} / \mathrm{mL})$ is shown by dashed line without correction. All spectra were recorded using a cuvette of $0.105-\mathrm{mm}$ path length.

this study are compatible with those previously reported using other techniques. Dynamic light scattering yielded hydrodynamic radii of $23 \pm 3,28 \pm 3$, and $31 \pm 3 \AA$ for the $\mathrm{OG}$ micelles at concentration of 1,2 , and $5 \%(\mathrm{w} / \mathrm{v})$, respectively (18). Similar measurement revealed a range of molecular weight from 25000 to 36000 , depending on the temperature, for the OG micelles at low concentration $(\sim 1 \%)(19)$. The molecular weights were also confirmed by a combination of sedimentation velocity and diffusion experiments (18). A somewhat smaller value of $R_{\mathrm{g}}=17.7 \AA$ was obtained for $1 \%(\mathrm{w} / \mathrm{v}) \mathrm{OG}$ aqueous solution from another SANS measurement (20).

Sizes of the Intact and Reconstituted B820 Subunits. Structural stability and homogeneity of the samples are essential for the SANS measurement. A small amount of large aggregates in the highly concentrated solution could generate strong scattering in the small-angle region and consequently bring about significant errors in the following evaluation process. Since the absorption spectrum is particularly sensitive to the aggregation and configuration of pigment molecules in the B820 subunits, it was used as a probe to ensure that our samples remained homogeneous during the measurements. Figure 2 shows the absorption spectra of the intact B820 subunit prepared by dissociation of carotenoid-depleted LH1 complex and the reconstituted B820 subunit from apopolypeptides and BChl $a$. Due to the difference in sample concentration, the absorption spectrum of intact B820 subunit was adjusted to have the same intensity as that of reconstituted B820 subunit at $819 \mathrm{~nm}$. The two spectra obtained with different preparations almost overlapped, indicating that both the B820 subunits have essentially the same structure. It was confirmed from the spectroscopic homogeneity that no higher aggregates existed after centrifuging the solutions even at high concentrations (in the millimolar range). All samples were stable at room temperature during the SANS data acquisition, typically 


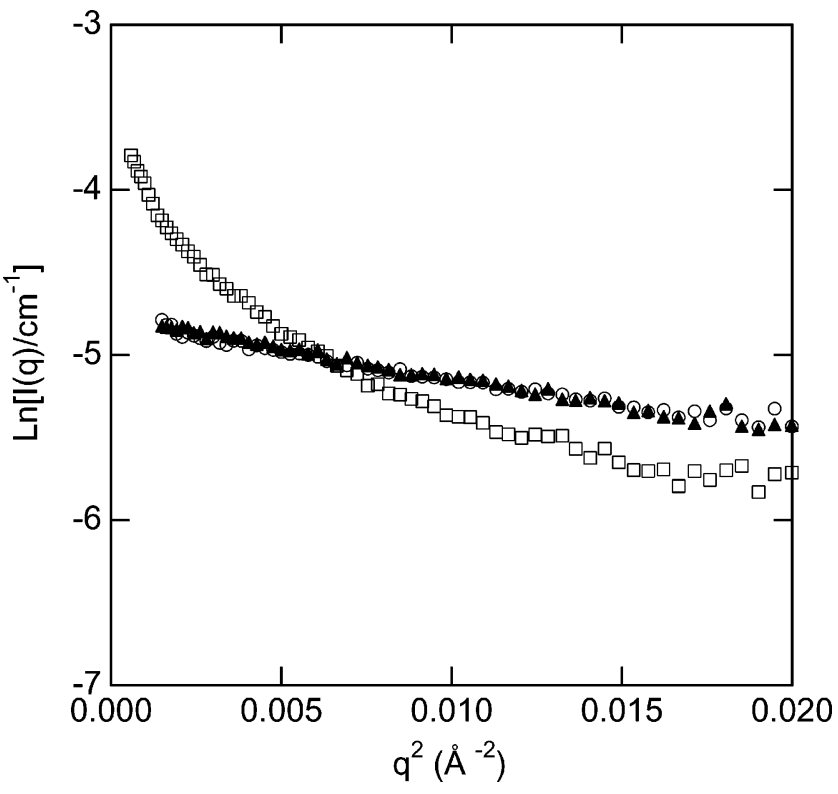

FIGURE 3: Guinier plots of SANS profiles for the intact B820 subunit $(\mathbf{\Delta})$, reconstituted $\mathrm{B} 820$ subunit $(\mathrm{O})$ and apopolypeptide $(\square)$ at OG- $d_{28}$ concentration of $4 \%(\mathrm{w} / \mathrm{v})$. The absolute scattering intensities were normalized by sample concentrations.

$1 \mathrm{~h}$, as there was no essential change in the absorption spectra after measurements.

Guinier plots of the SANS profiles from both intact and reconstituted B820 subunits in $4 \%(\mathrm{w} / \mathrm{v})$ OG- $d_{28}$ solutions are shown in Figure 3. The scattering intensities were collected with a sample-to-detector distance of $2 \mathrm{~m}$ and were normalized by sample concentrations. The two sets of Guinier plots were completely identical and can be fitted with a single straight line over a wide $q$ range, indicating that both the samples are indeed homogeneous and can be characterized by one set of Guinier parameters. From eq 1 , the radius of gyration was determined to be $11.0 \pm 1.0 \AA$ for the $\mathrm{B} 820$ subunits. The molecular weight of the whole B820 complex calculated from eq 2 was $11400 \pm 500$, corresponding to one minimal unit consisting of one pair $\alpha \beta$-polypeptides and two BChl $a$ molecules. The result is in good agreement with the spectroscopic evidence that the absorption band at 820 $\mathrm{nm}$ arises from exciton interaction between two $\mathrm{BChl} a$ molecules in a heterodimer of $\alpha$ - and $\beta$-polypeptides $(4,5)$. No other components of higher aggregates were observed for the highly concentrated B820 solutions from the SANS experiments. Since both intact and reconstituted B820 subunits exhibited essentially the same values for the radius of gyration and molecular weight, they can be considered to have the same structure and conformation in the OG solutions.

SANS Analysis of LH1 Apopolypeptides. The LH1 apopolypeptides are a mixture of $\alpha$ - and $\beta$-polypeptides prepared by extracting all pigment molecules from the LH1 complex. The resultant absorption spectrum was characterized by a single band at $280 \mathrm{~nm}$ as shown in Figure 2. Guinier plot of SANS profile from the apopolypeptides dissolved in $4 \%$ $(\mathrm{w} / \mathrm{v})$ OG- $d_{28}$ is given in Figure 3 for a comparison with those of B820 subunits. The most remarkable feature is that the Guinier plot for the apopolypeptides exhibited a curvature in the small-angle region and cannot be fitted by a single set of Guinier parameters. The result clearly indicates that sizes of the pigment-depleted apopolypeptides are largely

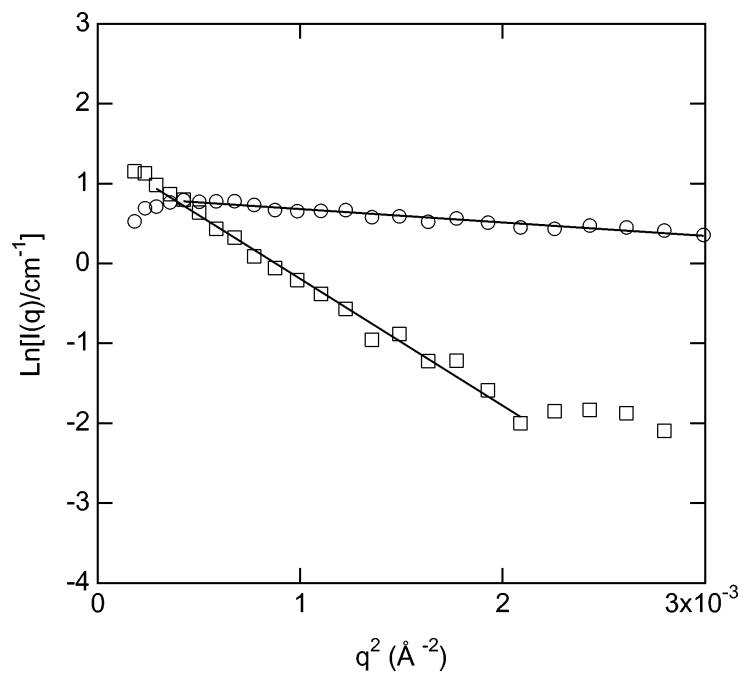

FIGURE 4: Guinier plots of SANS profiles for the separated $\alpha$-polypeptides $(O)$ and $\beta$-polypeptides $(\square)$ at $O G$ - $d_{28}$ concentration of $4 \%(\mathrm{w} / \mathrm{v})$. The straight lines were used for fitting the Guinier equation to the scattering data in small-angle region.

inhomogeneous in solutions at OG and polypeptide concentrations similar to those for the B820 subunits. From a multicomponent analysis using the Guinier equation, radii of gyration of the apopolypeptides were calculated in a range from 15 to more than $30 \AA$, which were much larger than those of the B820 subunits. The large values of $R_{\mathrm{g}}$ imply that apopolypeptides in OG solutions are not stable in the monomeric form and tend to form various high aggregates in the absence of BChl $a$ molecules. Thus, the SANS result provides evidence that $\mathrm{BChl} a$ molecules play a crucial role in stabilizing and maintaining the B820 subunits in a homogeneous dimeric state in the solutions.

SANS Behavior of Individual $\alpha$ - and $\beta$-Polypeptides. The tendency of apopolypeptides to form high aggregates in OG solutions can be considered as a consequence of complex interactions among the same and different polypeptide chains. To investigate the aggregation properties of individual polypeptides, we measured SANS profiles for the separately isolated $\alpha$ - and $\beta$-polypeptides in $4 \%$ OG- $d_{28}$ solutions. Figure 4 shows the Guinier plots in the small-angle region for the two polypeptides without being normalizd by polypeptide concentrations. The experiments were conducted with a sample-to-detector distance of $2 \mathrm{~m}$. Apparently, there was a significant difference between the sizes of $\alpha$ - and $\beta$-polypeptides. The radii of gyration and molecular weights were respectively calculated to be $12.1 \AA$ and 7010 for $\alpha$-polypeptide, and $69 \AA$ and 30240 for $\beta$-polypeptide. Taking into account about $15 \%$ errors in the SANS experiment and evaluation by the Guinier approximation, we conclude that the $\alpha$-polypeptides were uniformly dissolved in OG solution in a monomeric form whereas the $\beta$-polypeptides associated into large aggregates under the same experimental conditions. The concentration of $\alpha$-polypeptide $(16.6 \mathrm{mg} / \mathrm{mL})$ used in this study was more than double that for the $\beta$-polypeptide $(6.7 \mathrm{mg} / \mathrm{mL})$. Even at such high concentration, the $\alpha$-polypeptide still existed as a monomer at $4 \%(\mathrm{w} / \mathrm{v}) \mathrm{OG}$, whereas the $\beta$-polypeptide formed aggregates at a much lower polypeptide concentration but at the completely same OG concentration. Therefore, for the first time the experiments show that the $\beta$-polypeptide prefers an aggregation state more than the $\alpha$-polypeptide under the 
same experimental conditions (at the same polypeptide and OG concentrations). The result provides an important clue for the explanation of different behaviors of $\alpha$ - and $\beta$-polypeptides in reconstitution with $\mathrm{BChl} a$ molecules (see Discussion).

\section{DISCUSSION}

Determination of the size of membrane proteins in the presence of detergent always needs to take into account the effect of micelles formed by the detergent molecules. Hence, it is essential to appropriately evaluate the micelle sizes of the detergent itself in a systematic way. As shown in this study, the OG micelles have molecular weights of about 30000 to 50000 , corresponding to aggregation numbers of about 100 to 170 over a concentration range from 1 to $5 \%$ $(\mathrm{w} / \mathrm{v})$. In contrast, the real size of B820 subunit in $4 \%(\mathrm{w} / \mathrm{v})$ OG solution is actually much smaller than that of the OG micelles at the same OG concentration. Although we do not precisely know how many OG molecules attach to one B820 subunit, the number of attached OG could be of the same order of magnitude, if not equal, to the aggregation number of micelles formed by OG alone because the LH1 is considered to be a highly hydrophobic integral membrane protein complex. The large molecular weight of B820containing micelles is also evident from the broad line widths observed in nuclear magnetic resonance spectroscopy (13). The result may explain why there have been large discrepancies in estimation of the size of B820 subunit using the conventional gel exclusion chromatography. The micelle size was also found to strongly depend on the detergent used. Another nonionic detergent, $n$-heptyl $\beta$-D-thioglucopyranoside, has molecular structure, molecular weight, and critical micelle concentration similar to those of OG and can be used for preparing high-quality B820 subunits (data not shown). However, this detergent was shown by our SANS analysis to have a much larger micelle size than $\mathrm{OG}$ with a radius of gyration of $52 \AA$ and molecular weight of about 70000 at concentration of $5 \%(\mathrm{w} / \mathrm{v})$.

Both the intact B820 subunit prepared by dissociation of LH1 complex and the reconstituted B820 subunit prepared from separate apopolypeptides and $\mathrm{BChl} a$ were highly homogeneous and stable at high concentrations (in millimolar range) and room temperature. The SANS results of this study provide direct and independent evidence for the intact B820 subunit as a heterodimer consisting of one pair of $\alpha \beta$ polypeptides and two $\mathrm{BChl} a$ molecules. This is also true for the reconstituted B820 subunit because it was prepared in a stoichiometric ratio and its scattering profile was completely the same as that of the intact B820 subunit after normalization by concentration. The $\beta$-polypeptide alone was shown to be able to form a 820-nm-absorbing complex with $\mathrm{BChl} a$, probably in a $\beta_{2}(\mathrm{BChl} a)_{2}$ form, whereas the $\alpha$-polypeptide alone can only remain as $\mathrm{BChl} a$-bound monomers $(2,8)$. Our result reveals that the $\alpha \beta(\mathrm{BChl} a)_{2}$ form is predominant in the reconstituted B820 subunits, provided all components are present in a stoichiometric ratio, and the $\beta_{2}(\mathrm{BChl} a)_{2}$ form and $\mathrm{BChl} a$-bound $\alpha$-polypeptides, if they exist, can be ignored. The strong preference also coincides with the result of association constant measurements, which showed that mixed $\alpha$ - and $\beta$-polypeptides had an association constant about 60 times larger than the $\beta$-polypeptides alone in the presence of $\mathrm{BChl} a(21)$.
Since the neutron scattering length density of perdeuterated OG is almost identical with that of $100 \% \mathrm{D}_{2} \mathrm{O}$, we were able to selectively extract scattering from the B820 subunit without perturbation from the attached detergent. This allowed an accurate evaluation of the size of the B820 complex. In principle, the radius of gyration determined from the SANS experiment can also be calculated from the molecular structure. However, because no high-resolution structure is available for the LH1 complex, here we use the minimal structural unit of the LH2 complex from photosynthetic bacteria as an analogue for comparison between the measured and calculated radius of gyration. Calculations were conducted by program CRYSON $(22,23)$ using the atomic coordinates of two LH2 structures (Protein Data Bank accession numbers: $1 \mathrm{KZU}$ and $1 \mathrm{LGH}$ ) from Rhodopseudomonas (Rps.) acidophila and Rsp. molischianum $(24,25)$. Under the experimental condition of this study, the radii of gyration were calculated to be 17.4 and $18.8 \AA$ for the minimal units of LH2 complexes from Rps. acidophila and Rsp. molischianum, respectively. These values were larger than that of the B820 subunit obtained from SANS measurement. The result may be interpreted by assuming that $\alpha \beta$-polypeptides and $\mathrm{BChl} a$ molecules in the B820 subunit are packed in a more compact way compared with those in the minimal unit of LH2 complex or, alternatively, that much different conformations are adopted for the polypeptides. The compact packing of B820 could be considered as a factor responsible for the larger red shift of LH1 $Q_{y}$ band, while the possibility of different conformations has recently been suggested by NMR studies $(26,27)$. The $\beta$-polypeptide of LH1 complex from Rhodobacter sphaeroides was shown to have a more bent conformation than that of LH2 complex in both organic solvent and detergent solution, comprising two $\alpha$-helical regions joined by a flexible central hinge. Whether such a bent structure reflects its nature in vivo remains unclear.

It is of interest to note from the SANS results that the BChl $a$-depleted LH1 $\alpha \beta$-polypeptides showed a highly inhomogeneous behavior in the detergent solution. This feature highlights pigment-pigment and pigment-polypeptide interactions as the predominant association forces between the $\alpha$ - and $\beta$-polypeptides. Similar situation was revealed from the structures of LH2 complex, where helixhelix interaction was lacking and extensive mediation of structural contacts was largely via the pigment molecules within the transmembrane region $(24,28)$. The heterogeneity in solution can also be understood by different aggregation properties between the $\alpha$ - and $\beta$-polypeptides. The radii of gyration determined by the SANS measurements were 12.1 and $69 \AA$ for the $\alpha$ - and $\beta$-polypeptides, respectively. On the basis of the NMR structures of LH1 $\beta$-polypeptide of Rhodobacter sphaeroides $(26,27)$, the radii of gyration were calculated to be $16.1 \AA$ for the most bent conformation and $19.4 \AA$ for a more extended conformation. The results indicate that the $\alpha$-polypeptide remained as a monomer in OG solution, whereas the $\beta$-polypeptide formed large aggregates. The different properties in association may have influence on the ability to form a functional subunit with $\mathrm{BChl} a$. It was shown that the $\beta$-polypeptide alone can form a complex with BChl $a$ characterized by a red-shifted absorbance at $820 \mathrm{~nm}$, whereas $\alpha$-polypeptide alone was unable to form any red-shifted complex with the pigments (2). The capability of $\beta$-polypeptide to form the $820-\mathrm{nm}$ - 
absorbing complex, probably with a $\beta_{2}(\mathrm{BChl} a)_{2}$ structure, may be related to its nature of self-association. However, in the presence of both $\alpha$-polypeptide and BChl $a$, the $\beta$-polypeptide strongly favors to form a heterodimeric complex. Thus, formation of the uniform B820 subunit is a consequence of complex interactions between the two polypeptides and BChl $a$ molecules. We are currently investigating the structural basis for the self-association of $\beta$-polypeptide in detergent solution and the roles of $\alpha$-polypeptide and BChl $a$ in weakening such interaction to maintain the heterodimeric structure.

\section{REFERENCES}

1. Miller, J. F., Hinchigeri, S. B., Parkes-Loach, P. S., Callahan, P. M., Sprinkle, J. R., Riccobono, J. R., and Loach, P. A. (1987) Biochemistry 26, 5055-5062.

2. Parkes-Loach, P. S., Sprinkle, J. R., and Loach, P. A. (1988) Biochemistry 27, 2718-2727.

3. Mechenstock, R., Brunisholz, R. A., and Zuber, H. (1992) FEBS Lett. 311, 128-134.

4. van Mourik, F., van der Oord, C. J. R., Visscher, K. J., ParkesLoach, P. S., Parkes, P. A., Visschers, R. W., and van Grondelle, R. (1991) Biochim. Biophys. Acta 1059, 111-119.

5. Visschers, R. W., Chang, M. C., van Mourik, F., Parkes-Loach, P. S., Heller, B. A., Loach, P. A., and van Grondelle, R. (1991) Biochemistry 30, 5734-5742.

6. Pandit, A., Visschers, R. W., van Stokkum, I. H. M., Kraayenhof, R., and van Grondelle, R. (2001) Biochemistry 40, 12913-12924.

7. Arluison, V., Seguin, J., and Robert, B. (2002) FEBS Lett. 516, 40-42.

8. Arluison, V., Seguin, J., and Robert, B. (2002) Biochemistry 41, $11812-11819$.

9. Hunt, J. F., McCrea, P. D., Zaccai, G., and Engelman, D. M. (1997) J. Mol. Biol. 273, 1004-1019.

10. Tiede, D., and Thiyagarajan, P. (1996) in Biophysical Techniques in Photosynthesis (Amesz, J. a. H., A. J., Ed.), pp 375-390, Kluwer Academic Publishers, The Netherlands.

11. Wang, Z.-Y., Umetsu, M., Yoza, K., Kobayashi, M., Imai, M., Matsushita, Y., Niimura, N., and Nozawa, T. (1997) Biochim.
Biophys. Acta 1320, 73-82.

12. Gall, A., Dellerue, S., Lapouge, K., Robert, B., and BellissentFunel, M.-C. (2001) Biopolymers 58, 231-234.

13. Wang, Z.-Y., Muraoka, Y., Shimonaga, M., Kobayashi, M., and Nozawa, T. (2002) J. Am. Chem. Soc. 124, 1072-1078.

14. Wang, Z.-Y., Shimonaga, M., Muraoka, Y., Kobayashi, M., and Nozawa, T. (2001) Eur. J. Biochem. 268, 3375-3382.

15. Pilz, I. (1969) J. Colloid Interface Sci. 30, 140-144.

16. Jacrot, B., and Zaccai, G. (1981) Biopolymers 20, 2413-2426.

17. Guinier, A., and Fournet, G. (1955) Small Angle Scattering of $X$-rays, John Wiley, New York.

18. Lorber, B., Bishop, J. B., and DeLucas, L. J. (1990) Biochim. Biophys. Acta 1023, 254-265.

19. Kameyama, K., and Takagi, T. (1990) J. Colloid Interface Sci. 137, $1-10$.

20. Thiyagarajan, P., and Tiede, D. (1994) J. Phys. Chem. 98, 1034310351.

21. Meadows, K. A., Parkes-Loach, P. S., Kehoe, J. W., and Loach, P. A. (1998) Biochemistry 38, 3411-3417.

22. Svergun, D. I., Richard, S., Koch, M. H. J., Sayers, Z., Kuprin, S., and Zaccai, G. (1998) Proc. Natl. Acad. Sci. U.S.A. 95, 22672272.

23. Svergun, D. I., Barberato, C., and Koch, M. H. J. (1995) J. Appl. Crystallogr. 28, 768-773.

24. McDermott, G., Prince, D. M., Freer, A. A., HawthornthwaiteLawless, A. M., Papiz, M. Z., Cogdell, R. J., and Isaac, N. W. (1995) Nature 374, 517-521.

25. Koepke, J., Hu, X., Muenke, C., Schulten, K., and Michel, H. (1996) Structure 4, 581-597.

26. Conroy, M. J., Westerhuis, W. H. J., Parkes-Loach, P. S., Loach, P. A., Hunter, C. N., and Williamson, M. P. (2000) J. Mol. Biol. 298, 83-94.

27. Sorgen, P. L., Cahill, S. M., Krueger-Koplin, R. D., KruegerKoplin, S. T., Schenck, C. C., and Girvin, M. E. (2002) Biochemistry 41, 31-41.

28. Prince, S. M., Papiz, M. Z., Freer, A. A., McDermott, G., Hawthornthwaite-Lawless, A. M., Cogdell, R. J., and Isaac, N. W. (1997) J. Mol. Biol. 268, 412-423.

BI034436D 